\title{
TEORÍA DE LA COMUNICACIÓN Y EPISTEMOLOGÍA
}

\author{
EDISON OTERO BELLO*
}

I

Un manejo siquiera panorámico de la investigación y la reflexión en el área de la comunicación entre, digamos, las décadas de los ' 40 y los ' 80 , puede sugerir al estudioso algunas primeras conclusiones provisionales.

Entre las más socorridas están las siguientes:

1. El área se caracteriza por una cantidad insuficiente de investigación. Hace falta, pues, más investigación.

2. El área se caracteriza por una diversidad interpretativa (modelos, hipótesis, enfoques, etc.) que no ha podido ser integrada en una formulación única.

Aunque tienen apariencia neutral, inocente y estrictamente diagnóstica, ambos juicios se sustentan en epistemologías implícitas.

Por de pronto, la afirmación sobre el monto insuficiente de investigación supone conocer el monto que sí resultaría suficiente; y supone que ese monto suficiente, una vez alcanzado, obrará la consecuencia de dar la base para la teoría madura que iluminará el área y será referencia creíble como conocimiento. Este razonamiento, sin duda, es positivista y cree que el conocimiento se construye inductivamente a partir de los datos observados. Se puede sostener, por ende, que nuestro juicio 2 se infiere o es la consecuencia lógica del juicio 1: la diversidad interpretativa es el resultado ineludible de la insuficiencia investigacional. En algún momento del futuro, más próximo o más lejano, los datos reunidos por la investigación constituirán una "masa crítica" suficiente para generar teoría verdadera. Investigar más seguiría estando entonces, lógicamente, a la orden del día, sólo que las cosas no parecen ser tan simples.

Por de pronto, hay quienes afirman que el conjunto de la variada investigación tempírica, de laboratorio, de campo) permite sacar conclusiones; lo creyeron así Joseph Klapper (1960), y Bernard Berelson (1964), hace ya tres décadas y tanto, convencidos de que era posible formular algunas generalizaciones sólidas.

Pero existe otra razón de peso para considerar que las cosas no son tan simples: la epistemología positivista no es la única epistemología e incluso más, se trata de una zoncepción del desarrollo de las ciencias que ha sido cuestionada severamente (Hanson 1958, Kuhn 1962, Popper 1956, 1962, 1972, Feyerabend 1975, Lakatos 1978).

¿Cómo comprender, entonces, el agua que ha pasado bajo el puente entre los años " 40 y los años ' 80?, ¿es la teoría de la comunicación una auténtica disciplina, cumpliendo su fase "preparadigmática", para usar terminología de Th. Kuhn?; ¿o no es una disciplina ino simplemente un área, como lo sostuvo W. Schramm (1959), zona de cruce y sonfluencia de otras disciplinas?

\footnotetext{
* Director Escuela de Ciencias Sociales, Facultad de Ciencias Sociales, Universidad de Chile.
} 


\section{REVISTA DE SOCIOLOGÍA}

Pueden formularse muchas preguntas más, preguntas que es preciso hacerse. El camino de "más investigación, más investigación" puede llegar a parecerse bastante a una marcha ciega si no tiene una dirección o no se hace en función de un programa o alguna conjetura. Por fuerte que pueda parecer el juicio, nos parece que la necesidad investigacional - siendo ineludible - no es el punto central. La cuestión central radica, a nuestro parecer, en la insuficiente profundización teórica. Una proporción significativa de la literatura - e incluso de aquella que se considera obligada- adolece de una franca pobreza intelectual, sorprendentemente desconocedora de referencias teóricas, singularmente aislada de tradiciones (científicas, filosóficas), como si no tuviera que dar cuenta sino de sí misma.

La necesidad de teorización de profundidad creciente exige, por de pronto, una relectura, una reinterpretación desde los orígenes mismos, poniendo a la vista, más que las conclusiones expresas, las adhesiones conceptuales no explícitas, las preferencias intelectuales subterráneas o de fondo, las adhesiones temáticas - para decirlo con categorías de G. Holton (Holton 1963). Contrariamente a lo que muchos balances o "estados del arte" declaran, la historia de las ideas en comunicación todavía está por pensarse auténticamente, aplicándose sobre ella reflexión fina, dedicada, sostenida. Muchas exposiciones, interpretaciones y lecturas más o menos autorizadas sobre Laswell, Lazarsfeld, McLuh $\mathbf{n}$, Bandura o Eco no traspasan el nivel de la divulgación esquemática, la trivialización o la mera referencia; con frecuencia, ese material da la impresión de ser cuarta o quinta lectura, con ese dejo de impostura que tiene indefectiblemente la ausencia de un contacto directo con las fuentes (mayormente, los textos originales).

\section{II}

En una primera aproximación, parece sostenible afirmar que hay signos de que una reflexión como la que hemos bosquejado está en sus primeros pasos. Es nuestro propósito examinar aquí la propuesta desarrollada por Abraham Nosnik (1991) que, en lo sustantivo, quiere evaluar el trabajo científico en comunicación social usando el modelo de la metodología de los programas de investigación científica de Imre Lakatos. Se trata de una propuesta digna de análisis porque, ante todo, no es común ni habitual en el área que se abandonen las prácticas autistas, autorreferenciales y se arriesgue poner en convergencia dos líneas de trabajo por lo general sin zonas de contacto. La propuesta de Nosnik plantea un cruce de epistemología y teoría de la comunicación.

Decimos "un" cruce porque no es el único posible; de hecho, la evolución del área puede ser abordada desde las ideas de Kuhn (ciencia normal — anomalía-revoluciónnuevo paradigma), las Popper (conjetura-falsación) las del propio Lakatos, las de Feyerabend (multiplicidad de métodos, proliferación de teorías) o las de Gerald Holton (análisis temático), entre otros asedios posibles.

Nosnik ha optado por la metodología de los programas de investigación científica. Al hacerlo, asume como unidad de análisis el "programa de investigación", renunciando por ello a otras unidades de análisis planteadas en epistemología: un juicio particular, una teoría, una red de teorías, un paradigma o un par temático, por ejemplo.

En uno de sus textos clásicos, Lakatos presenta así su postura: "Uno de los aspectos cruciales del falsacionismo sofisticado es que sustituye el concepto de teoría, como concepto básico de la lógica de la investigación, por el concepto de series de teorías. Lo que ha de ser evaluado como científico o pseudocientífico es una sucesión de teorías y no una teoría dada. Pero los miembros de tales series de teorías normalmente están relacio- 
nados por una notable continuidad que las agrupa en programas de investigación. Esta continuidad (reminiscente de la ciencia normal de Kuhn), juega un papel vital en la historia de la ciencia; los principales problemas de la lógica de la investigación sólo pueden analizarse de forma satisfactoria en el marco suministrado por una metodología de los programas de investigación". (Lakatos, 1970).

Como se sabe, Lakatos distinguía entre programas progresivos y regresivos, y fundaba la distinción en el desarrollo de predicciones nuevas e inesperadas y la corroboración de, al menos, algunas de tales predicciones nuevas. Todavía más, un programa de investigación (una serie de.teorías en desarrollo) tiene una estructura: un núcleo central firme y persistente, una heurística (técnica para la solución de problemas) y un cinturón protector de hipótesis auxiliares (Lakatos 1976).

De la mano del lakatosianismo, Nosnik identifica como programas de investigación científica en el área de la comunicación las siguientes:

1. "El programa de efectos poderosos de los medios de comunicación social.

2. El programa de efectos limitados de los medios de comunicación social.

3. El programa de usos y gratificaciones de los medios de comunicación social.

4. El programa de "consefectos" de los medios de comunicación social.

5. El programa de efectos de los medios de comunicación contingentes de la audiencia" (Nosnik, 1991, pág. 73).

El primer programa aparece asociado a la fórmula clásica de Laswell y el cinturón protector auxiliar estaría constituido por las hipótesis de la función de socialización de los medios masivos, la de efectos de brechas del conocimiento (gap hypothesis), la agenda-setting, la de los efectos de los formatos de los medios y la hipótesis de los efectos ideológicos de los medios masivos. Nosnik presenta también estas hipótesis como cinco creencias principales:

1. "que los medios masivos tienen una función de socialización en diferentes poblaciones;

2. que los efectos de los medios masivos pueden dividir a sus audiencias en sectores alta y pobremente informados;

3. que los medios masivos ejercen una función de jerarquización de la información sobre sus audiencias.

4. que la presencia misma de distintos formatos en los diferentes medios influye en la transmisión de los mensajes causando, a su vez, efectos diferenciales sobre sus audiencias, $y$

5 que los medios masivos transmiten e imponen distintas ideologías (es decir, valores, expectativas, modos de vida, etc.) sobre sus audiencias" (Nosnik 1991, pág. 78).

El segundo programa (de los efectos limitados) está asociado a las investigaciones de Paul Lazarsfeld, Elihú Katz y Joseph Klapper, entre otros. En lo sustantivo, sostienen la operación de factores intermediadores entre los medios de comunicación y sus audien¿ias; los medios serían una variable interviniente entre otras. Entre las hipótesis auxiliares lel programa estaría la idea de selectividad: exposición selectiva, percepción selectiva y : etención selectiva; otras serían la influencia del grupo primario, la función de los líderes ntermediarios y las relaciones interpersonales (Katz, y Lazarsfeld, 1955).

Sobre el tercer programa, Nosnik se limita a una referencia muy limitada, afirmando que se trata de una postura de reciente génesis que sostiene la existencia de efectos 
recíprocos entre los medios y sus receptores. Este programa aparece asociado, principalmente, al nombre del investigador sueco S. Windahl (Windahl, 1981).

El quinto programa, de los efectos contingentes, es asociado por Nosnik a los nombres de Melvin Defleur y Sandra Ball-Rokeach (1975).

Este programa sostiene la necesidad de comprender la influencia de los medios en una relación de mutua interdependencia con el sistema social y la audiencia. En lo específico, los autores afirman que los medios cumplen funciones de transmisión de información y que hay condiciones sociales en que esa función se vuelve crucial, porque se incrementa la necesidad de información de las personas; estas condiciones peculiares coincidirían con épocas de crisis social. En tales casos, se produciría un aumento del índice de dependencia de las personas respecto de los medios en tanto generadores de información. Como hipótesis auxiliares pueden identificarse las ideas de efectos cognitivos, cambios afectivos, activación y desactivación.

Hemos dejado el cuarto programa - el de usos y gratificaciones- para el final de esta enumeración muy sucinta, apoyados en la evidente mayor dedicación que Nosnik le dedica, intentando con él una reconstrucción racional lakatosiana.

\section{III}

En el capítulo 4, dedicado a examinar en detalle el programa de Usos y Gratificaciones, Nosnik aclara que habla de él "tanto como un programa científico de investigación, como parte del cinturón de protección de los programas de investigación de efectos poderosos y limitados" (1991, pág. 94). Luego de referir tres períodos en el desarrollo del programa, Nosnik identifica su núcleo sosteniendo que mientras que "por un lado, los programas tradicionales de efectos de los medios están interesados en qué es lo que los medios le hacen a la gente, los investigadores de Usos y Gratificaciones están preocupados por qué es lo que la gente hace con los contenidos de los medios" (1991, pág. 96).

Como autores asociados al programa, Nosnik señala a Joseph Klapper, J.G. Blumer y Elihú Katz, entre otros. Hablando de modo general, el enfoque de usos y gratificaciones concibe a la audiencia como una entidad dinámica, no pasiva, formada por miembros que, en el propósito de satisfacer necesidades, eligen, entre otras fuentes, los medios de comunicación. En el aspecto total de las necesidades, sólo una parte es cubierta (y en grados variantes) por la comunicación social. Además de la gratificación de necesidades resultante de la exposición a los medios de comunicación, esta exposición tendría otro tipo de consecuencias, en su mayor parte no deseadas.

El siguiente conjunto común de supuestos será característico del enfoque de usos y gratificaciones:

1. La utilización de los medios de comunicación está dirigida hacia metas. Utilizamos los medios masivos para satisfacer necesidades específicas. Estas necesidades surgen de nuestro medio ambiente social.

2. Los receptores seleccionan los tipos de medios y el contenido de los medios para satisfacer sus necesidades. Así, la audiencia inicia el proceso de comunicación masiva, y somos capaces de "inclinar los medios" a nuestras necesidades más fácilmente de lo que los medios nos pueden supeditar.

3. Existen otras fuentes de satisfacción de necesidades y los medios masivos deben competir con ellas. Algunas fuentes de satisfacción distintas de los medios son la 
familia, los amigos, la comunicación interpersonal, otras actividades en el tiempo libre, dormir y las drogas.

- La audiencia está consciente de sus necesidades y puede informar sobre ellas cuando se le pregunta. Los miembros de la audiencia también están conscientes de sus razones para usar los medios masivos (Tan, 1981).

I mo es obvio, este planteamiento implica o requiere ineludiblemente algún tipo de $\therefore$ sificación de necesidades; es lo que Katz, Gurevitch y Haas han ofrecido como tal, :nsando las necesidades en relación con los medios: necesidades cognoscitivas (inforación, conocimiento y comprensión), afectivas (experiencias estéticas, placenteras y mocionales), integrativas en lo personal (credibilidad, confianza, estabilidad, status), -tegrativas en lo social (familia, amigos, grupos) y escapistas (diversión y alivio de insiones) (1973).

No es nuestro propósito entrar en el detalle del examen reconstructivo que Nosnik - lica al programa de usos y gratificaciones, al que considera, en general, como teórica.ente progresivo. Lo que pretendemos, más bien, es un primer juicio sobre la propuesta Tosnik de aplicar la metodología de Lakatos a la comunicación social.

De acuerdo a su planteamiento, como ya hemos visto esquemáticamente, competirían el área cinco programas de investigación: el de los efectos poderosos, el de los efectos aitados, el de usos y gratificaciones, el de consefectos y el de efectos contingentes.

Resulta prácticamente inevitable preguntarse si esta diversidad que Nosnik presenta pueda ser objeto de un mayor esfuerzo de síntesis, aunque no por la mera necesidad síntesis y de simplificación.

Ya en una primera mirada, no hay cómo ocultar esta evidencia: de los cinco rogramas", cuatro de ellos aparecen identificados a través de un mismo concepto, el ncepto de "efectos". El propio Nosnik, incluso, avanza la idea de que el programa - tante (usos y gratificaciones) puede ser considerado también como formando parte del nturón protector del programa de efectos limitados. Si esto tiene fundamento, entonces - aún más ostensible la presencia del concepto "efectos". Podríamos afirmar, pues, y sin irnos del ámbito lakatosiano de interpretación, que estamos ante un solo programa: el grama de los efectos. Los otros serían variantes de su cinturón protector. Nuestra mmación es que, poderosos o limitados, de corto o largo plazo, emocionales o cogniti$\therefore$ retroalimentándose o no con sus usuarios, seguimos planteándonos en el ámbito de - efectos.

Esto genera una serie de reflexiones. Por una parte, podríamos decir que no ha habido $\because 0$ modelo de pensamiento en el área académica y de investigación y que, en rigor, la - estigación se pone en marcha históricamente en el entendido implícito de que los - ultados respaldarán inevitablemente la existencia de efectos de los medios de comu-ición.

Sólo que la historia de la investigación jamás ha respaldado al modelo de los efectos, menos en su versión más ortodoxa (efectos poderosos). Esta historia del área es, en esa - dida, una historia de frustraciones progresivas y, también, es la historia de la imposidad de avanzar más allá del modelo de los efectos.

Seguramente, lo que introduce una gran dosis de equívoco en el tema es la presencia Jesmentible de una creencia social en el poder de los medios de comunicación, anterior inicio estable de la investigación. En el año 1948, B. Berelson, por una parte, y - zarsfeld y Merton, por otra, lo aluden explícitamente en sendos artículos (Berelson 
1948; Lazarsfeld y Merton, 1948). Existe claridad sobre los adherentes y difusores de tal creencia: los encargados gubernamentales de propaganda, los anunciantes, los publicistas, los periodistas, los propietarios de medios, etc.

Podría pensarse que esta creencia y la investigación corren por líneas paralelas que no se juntan. Eso ocurre progresivamente, pero en los inicios la convergencia parece clarísima. Laswell, uno de los padres fundadores, sostiene, un par de años antes que Berelson, Lazarsfeld y Merton:

"Tal como se ha desarrollado en los pocos años pasados, el estudio científico de la comunicación se centra en torno de las cuatro fases sucesivas de todo acto de comunicación: ¿en qué canales tienen lugar las comunicaciones? ¿quién comunica? ¿qué es comunicado? ¿quién es afectado por la comunicación y cómo?" (Smith, Laswell, Casey, 1946).

Esta concepción, como es claro, da por hecho que hay efectos. El libro referido contiene 4 capítulos, cada uno de ellos dedicado a una de las fases; Laswell escribe aquellos dedicados a los contenidos y a los efectos.

Resulta más confirmatorio todavía el hecho que los 4 capítulos aludidos estén encabezados por el título "La Ciencia de la Comunicación de Masas: Cuatro Ensayos". O sea, estamos hablando de realidades cuya existencia está confirmada por la ciencia.

Sin embargo, cuando se examinan detenidamente los ensayos de Laswell, se desatan muchas dudas. Laswell clasifica los efectos en 5 tipos, los que asocia a 5 tipos de respuestas de la audiencia a los contenidos de las comunicaciones: atención, comprensión, disfrute, evaluación y acción. Se habla de efectos para referirse a la parte de la respuesta que está por encima del mínimo necesario para establecer una situación de comunicación. Sostiene Laswell, a continuación, que las respuestas de la audiencia están en función de las identificaciones, demandas y aceptaciones de sus miembros, las que, a su vez, son provocadas por dos series de factores: el entorno y las predisposiciones. Enseguida, Laswell afirma: "Cuando analizamos el contenido de la comunicación estamos describiendo uno de los factores que afectan la respuesta. Las identificaciones, demandas y aceptaciones de una persona son "causadas por" (son "funciones de") dos series de factores: el entorno y la predisposición. En este punto surge una cuestión técnica: ¿cómo distinguimos el "contenido de los medios" de las otras partes del entorno? Podemos contestar la pregunta cuando tenemos una teoría general del proceso de comunicación" (Laswell, 1946).

Aquí tenemos a los contenidos de los medios de comunicación como un factor entre otros. Y ello no se parece en nada a las "balas mágicas" o los "efectos hipodérmicos" que son asociados habitualmente al modelo laswelliano de las preguntas. Laswell ni siquiera se compromete teóricamente con el concepto de "masa". Habla de personas, grupos, audiencias, culturas.

¿Qué autor, entonces, puede ser identificado como el responsable de la formulación de efectos poderosos?

Si no se trata de Laswell (por las dudas que hemos expuesto), todavía menos podría tratarse de Paul Lazarsfeld o de Kurt Lewin, claramente en oposición a un modelo tal.

No sólo en el caso de Laswell sino también en el de Schramm, Klapper, Berelson, Katz y otros, se tiene la fuerte impresión de un uso inercial de los conceptos, de una utilización no asumida de las denominaciones. Se habla de "efectos" y de "masa", pero los desarrollos, los datos, las investigaciones, las conclusiones no se compadecen, en rigor, con el uso continuado de esos conceptos. Puede hablarse, tal vez, de negligencia 
intelectual. O, tal vez, de la fórmula de transacción entre un grupo de investigadores que se va abriendo paso y consideración, y una extendida creencia social que los rodea y con la que se topan habitualmente. ¿Por qué no entender así ese célebre párrafo de Klapper muchas veces citado?: "Profesores, sacerdotes, padres y legisladores nos han preguntado miles de veces, en los últimos quince años, si la violencia en los medios de comunicación produce delincuencia, si los medios aumentan o disminuyen el gusto del público y qué pueden hacer para la persuasión política de sus audiencias. Sobre estas cuestiones no sólo no hemos podido proporcionar respuestas definitivas sino que hemos hecho algo peor: hemos proporcionado evidencia para respaldar parcialmente cada punto de vista". (Klapper, 1957).

Todo esto daría fuerza a la tesis de algunos autores - que el propio Nosnik refiere-, ¿ gún los cuales la supuesta tradición de investigación sobre efectos poderosos jamás existió (Chaffee y Hochheimer, 1983). Si esto es así, la investigación en el área, aguijoneada por sectores sociales no asociados al mundo académico, debería ser caracte- izada como la creciente no corroboración de la creencia en los efectos poderosos. Como : sultado de estas sucesivas inferencias, terminaríamos por sostener que el único programa identificable es el de los efectos limitados, en cualquiera de sus versiones. Nosnik, - :cordémoslo, afirma que el programa de uso y gratificaciones significaría una ruptura : on el modelo de los efectos poderosos; caería, suponemos, bajo el concepto de efectos imitados.

\section{IV}

Resulta imprescindible, a estas alturas, recoger una afirmación que hemos hecho al - omienzo de este artículo. Llama poderosamente la atención, en la literatura del período $\therefore$ 'ue estamos encarando — de los ' 40 a los ' 80 - , la falta de profundidad teórica. En la ultitud de libros, antologías y artículos producidos en el período, rara vez surge un -abajo que abarque interrogantes sobre los conceptos que se usan y sobre los supuestos - by yacentes en las formulaciones, es decir, algún intento de tipo epistomológico destinaa metateorizar el modo de plantear los problemas. Hemos hablado de un uso "inercial" - los conceptos en la literatura referida, aludiendo con ello a una práctica intelectual que repara en los supuestos tras los conceptos que utiliza, tal vez por que se los tiene por - ¿quros y firmes y no cabría, entonces, abrirlos al proceso de la duda. Hay aquí alguna ase de confianza en la reflexión disponible, tal como se la comunica y reproduce.

Probablemente la falta de profundidad teórica era lo que Bernard Berelson, a fines de $\therefore$ años '50, tenía en mente al sostener que, después de la obra de los padres fundadores : la investigación en comunicación (Laswell, Lazarsfeld, Lewin y Hovland), la situación .. el área era de estancamiento y no se vislumbraba el desarrollo de ideas de igual calibre 3erelson, 1959).

Entre las réplicas al diagnóstico de Berelson está un artículo de Raymond Bauer, . ien se pregunta acerca de la necesidad de cambiar de paradigma para los estudios de :mpo en comunicación, dada la peculiar frustración experimentada en los esfuerzos por :erminar "efectos" (Bauer, 1959).

Ese mismo año, el sociólogo W. Phillips Davison afirma textualmente que "es $\therefore$ : masiado fácil pensar la comunicación en términos de causa y efecto, en términos de un ¿nsaje que es enviado desde su fuente a su destinatario y de la impresión o el impacto $\therefore$ resulta de su llegada. Esta visión del proceso de comunicación ha distorsionado y 
nulificado, en el pasado, un buen monto de investigación sobre los efectos de la comunicación" (Davison, 1959).

En la década siguiente, el sociólogo Eliot Freidson afirma que la orientación práctica de muchos estudios ha generado una notable laguna teórica en materia de comunicación, la que ha sido llenada por el mero sentido común. Entre las preocupaciones teóricas de Freidson está el concepto de "masa", y llega a sostener que se trata de un concepto inapropiado para definir el carácter de la audiencia de los medios de comunicación (Freidson, 1953).

Donald Roberts (Roberts, 1971) postula que el modelo causa-efecto ha sido el paradigma básico de la mayoría de los estudios sobre efectos de la comunicación. Señala, también, que ha habido muchas dificultades con la aplicación de este modo de ver las cosas. Sin embargo, a la hora de los balances, Roberts reitera la necesidad de, otra vez, pensar el problema de "los efectos".

Los autores que hemos citado constituyen excepción en la literatura del área; y, con todo, sin embargo, no logran llevar su incipiente reflexión epistemológica más allá de los límites de los conceptos que ellos mismos cuestionan. El resultado es una indesmentible inconsistencia en el discurso, una ambigüedad permanente en la argumentación, fruto de una teorización insuficiente. Un ejemplo reciente lo constituye un texto que se inscribe en la tendencia de interés creciente en la aplicación de métodos cualitativos de investigación en comunicación. Jensen y Jankowski, compiladores y también autores, reúnen textos que abundan en análisis de recepción, de audiencias, de contextos sociales, de comunidades, de aproximaciones históricas y etnográficas, de producción social de significados, etc., y, sin embargo, titulan su libro como un "Hanbook of Qualitative Methodologies for Mass Communication Research", en donde el concepto de "masa" continúa apareciendo, pero en franca inconsistencia con las posturas de la mayor parte de los trabajos del volumen (Jensen y Jankowski, 1991).

Los ejemplos pueden multiplicarse. El hecho a la vista es la insistencia en el uso de conceptos acerca de los cuales la reflexión no avanza más allá de unos primeros pasos. Visto por el reverso, revela, simultáneamente, la enorme fuerza inercial de un modo de pensar. Esto explica la situación de empantanamiento en que se halla la teoría de la comunicación, entendida como un área de trabajo y no como un producto teórico capaz de dar cuenta de su objeto. La única y valedera "revolución copernicana" que la reflexión comunicacional puede encarar es el abandono definitivo del modelo de los efectos, en cualquiera de sus versiones.

Este abandono la obligará a buscar nuevas categorías y a restablecer una fluida interacción con el conjunto de las ciencias sociales. Esta tarea, más que un problema de investigación de su objeto, es una tarea de reflexión sobre sí misma. Este tipo de reflexión tiene un nombre: se llama Epistemología.

\section{BIBLIOGRAFÍA}

Berelson, Bernard. Communications and Public Opinion, 1948, Incluido en Mass Communications, editado por Wilbur Schramm, The University of Illinois Press, 1949

Berelson, Bernard. The State of Communicactions Research, 1959. Public Opinion Quarterly, Vol. 223, No2.

Berelson, Bernard y Steiner, Gary. Human Behavior An Inventory of Scientific Findings. Harcourt, Brace \& World. New York. 1964.

Bader, Raymond. Comentario a Berelson, 1959. Public Opinion Quarterly, Vol. 23, N². 


\section{TEORÍA DE LA COMUNICACIÓN Y EPISTEMOLOGÍA}

$\checkmark$ W. PhrulPs. On the Effects of Communications, 1959. Public Opinion Quarterly, Vol. 23, No 3.

$:=4$ E.TD. PaLl. Contra el Método, 1975. Editorial Tecnos, 1981.

- Eliot. Communications Research and the Concept of Mass, 1953. Incluido en The Process and Effects of Mass C.mmunications, editado por Wilbur Schramm, 1971 .

- N.R. Patterns of Discovery. Cambridge University Press, 1961.

- G Gerald. Ensayos sobre el pensamiento científico en la época de Einstein, 1973. Alianza Editorial. 1982.

$\therefore$ K.B. y JANKOWSKI, N. A Handbook of Qualitative Methodologies for Mass Communications Research. Routledge, $\because 91$

E. GlRevitch, M. y HaAs, H. On the use of Mass Media for Important Things. American Sociological Review, $\because 3 . N^{\circ} 38$.

=... JosepH. The Effects of Mass Communication. The Free Press, New York, 1960.

- Thomas. La Estructura de las Revoluciones Cientificas, 1962. Fondo de Cultura Económica, 1971.

- Is. InRe. La Falsación y la Metodología de los Programas de Investigación Científica, 1970. Alianza Editorial, 983.

9. IMRE. ¿Por qué superó el programa de investigación de Copérnico al de Tolomeo?, 1976. Alianza Editorial, $9 \$ 3$.

is. IMre. La Historia de la Ciencia y sus Reconstrucciones Racionales, 1971. Alianza Editorial, 1983.

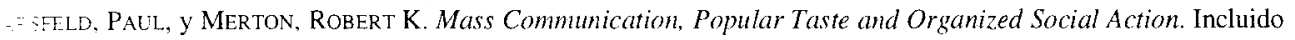
$\therefore$ The Communication of Ideas, editado por Lyman Bryson, Hasper \& Brothers, 1948.

- ABRAHAM. El desarrollo de la Comumicación Social: un Enfoque Metodológico. Editorial Trillas, 1991.

:- KARL. La Lógica de la Investigación Científica, 1959. Editorial Tecnos, 1967.

$\because:$ KARL. Conjeturas y Refutaciones. El Desarrollo del Conocimiento Científico, 1963. Editorial Paidos, 1967.

$\therefore$ KarL. Conocimiento Objetivo, 1972. Editorial Tecnos, 1974.

$\because$ - DONALD. The Nature of Communications Effects. Incluido en Schramm, W., 1971.

$\therefore$ … Wilbur. Comentario de Berelson, 1959. Public Opinion Quarterly, Vol. 23. N 2.

$\therefore$ B. Laswell., H. y CaSey, Ralph. Propaganda, Communication, and Public Opinion. Princeton University Press, 9.46.

AiExis, Mass Communications. Theories and Research. Grid Publishing Co. 1981.

$\therefore$ ¿. S. Uses and Gratifications at the Crossroads. Incluido en Mass Conmunications Review Yearbook, Vol. 2 S.ige, 1981. 